\title{
The potential gonadoprotective effects of grape seed extract against the histopathological alterations elicited in an animal model of cadmium-induced testicular toxicity
}

\author{
A.A. Morsi', L.M. Shawky², E.A. El Bana \\ ${ }^{1}$ Department of Histology and Cell Biology, Faculty of Medicine, Fayoum University, Fayoum, Egypt \\ 2Department of Histology and Cell Biology, Faculty of Medicine, Benha University, Benha, Egypt \\ ${ }^{3}$ Department of Anatomy, Faculty of Medicine, Benha University, Benha, Egypt
}

[Received: 3 November 2019; Accepted: 30 December 2019]

Background: Grape seed extract (GSE) is a powerful antioxidant containing high levels of bioflavonoids, vitamin $C$ and vitamin $E$. The aim of the work is to study the possible protective and ameliorative effects of grape seed extract in an animal model of cadmium (Cd)-induced testicular toxicity in rats.

Materials and methods: A thirty-day oral gavage study in adult male albino rats was performed using 32 animals, randomly divided into four equal groups; negative control, Cd (5 mg/k/day), GSE (100 mg/k/day), and Cd + GSE. Testicular weights were measured. Haematoxylin and eosin (H\&E) staining and proliferating nuclear cell antigen (PCNA) immunohistochemistry, as a marker for proliferation were done. Morphometric parameters were assessed and subjected to statistical analysis.

Results: The H\&E results showed atrophy and distortion of the seminiferous tubules (STS) with sloughing of the spermatogenic epithelium in cadmium group. The interstitial spaces were widened and showed oedema and mononuclear cell infiltrations. No remarkable changes were observed in the GSE-only group when compared to the control group. In Cd + GSE group, maintaining of the STs and their lining cells was evident. The immunohistochemical results showed marked positive PCNA immunoreactivity in both control and GSE groups, while negative immunoreaction was noticed in Cd group. Limited positive PCNA immunoreactivity was ameliorated in Cd + GSE group.

Conclusions: Grape seed extract protected against cadmium-induced testicular toxicity in rats, reducing induced histopathological changes, and maintaining testicular histoarchitecture. (Folia Morphologica 2020; 79, 4: 767-776)

Key words: cadmium, grape seed extract, testis, seminiferous tubules, proliferating nuclear cell antigen

\section{INTRODUCTION}

The burden of disease and death caused by environmental pollution is becoming a public health challenge worldwide, particularly in developing countries
[36]. Cadmium (Cd) is a heavy metal, globally spread environmental toxicant due to its varied industrial applications [17]. Apart from the occupational exposure of workers; diet is generally recognised as being the

Address for correspondence: Dr. A.A. Morsi, Department of Histology and Cell Biology, Faculty of Medicine, Fayoum University, Fayoum, Fayoum Governorate, Egypt, e-mail: ahmed_saqr4@yahoo.com 
main source of exposure to trace elements [15]. In its latest report, World Health Organization established a provisional tolerable monthly intake for $\mathrm{Cd}$ of $25 \mu \mathrm{g} / \mathrm{kg}$ body weight. It reported variable $\mathrm{Cd}$ concentrations in food but generally the kidney and liver of mammals and certain species of oysters, scallops, mussels, and crustaceans are major sources of $\mathrm{Cd}$. However, lower concentrations of $\mathrm{Cd}$ are found in vegetables, cereals, and starchy roots [35]. Tobacco smoking is another important source for $\mathrm{Cd}$ exposure [7]. About $1-3 \mu \mathrm{g}$ of $\mathrm{Cd}$ is absorbed by smoking one pack of cigarettes per day and, consequently, heavy smokers have more than double of the $\mathrm{Cd}$ body burden, compared to non-smokers [31].

Occupational exposure to $\mathrm{Cd}$ usually occurs during metal smelting or purifying, making batteries, and plastics. Atmospheric pollution with $\mathrm{Cd}$ could result from volcano activities on earth, human activities like melting and using fossil fuels, in addition to industrial processes such as smelting and refining of metals [24].

Cadmium toxicity is encountered in different body systems resulting in damage to various organs, particularly the testis, in both humans and animals [4]. Rodent testis, in particular, is sensitive to the toxic effects of $\mathrm{Cd}$ leading to severe testicular degeneration, seminiferous tubule damage and necrosis in rats [8].

Grape seed extract (GSE) is a natural product, recently identified to have an antioxidant property. GSE is a powerful antioxidant, which contains high levels of bioflavonoids, vitamin C and vitamin E [23]. The protective effect of GSE is attributed to regulating cell oxidative stress [18], reducing organ injury, improving the balance between oxidants and antioxidants [30], and reducing the release of inflammatory mediators [33]. In addition, anticarcinogenic effects have been reported [21].

Due to the toxic effects of $\mathrm{Cd}$ upon different body systems and organs including testis, the present work was designed to study the potential ameliorative and protective effects of a natural product, grape seed extract, against the testicular histopathological changes induced in an animal model of $\mathrm{Cd}$ intoxication. Most of the latest studies evaluated the $\mathrm{Cd}$ induced testicular toxicity, in regards to the degenerative and apoptotic changes seen in seminiferous tubules' epithelium. Herein, we focused on the proliferative capacity of spermatogenic cells, hence the efficiency of spermatogenesis, during the $\mathrm{Cd}$ toxicity and the effect of GSE on retaining such capacity.

\section{MATERIALS AND METHODS}

Animals

Thirty-two adult male albino rats, weighing about 200-250 g were used in the present study. The animals were locally bred in the animal house of Kasr El-Aini Faculty of Medicine, Cairo University, Egypt, housed at an ambient temperature of $27 \pm 1{ }^{\circ} \mathrm{C}$, maintained under a natural daily light/dark cycle and received free access to food and water ad libitum. For acclimatisation, the rats were handled manually for 1 week prior to the experiment. All ethical issues regarding animal handling and experimental procedures were followed and were compliant with the Guide for Care and Use of Laboratory Animals published by the United States National Institutes of Health [9]. Whenever possible, the procedures in the current study were conducted to avoid or minimise suffering, distress, and pain to animals.

\section{Drugs}

Cadmium, in the form of cadmium chloride $\left(\mathrm{CdCl}_{2}\right.$, $96 \%$ pure), was purchased from Sigma-Aldrich Corporation of industrial chemistry and biotechnology (St. Louis, Missouri, USA). Grape seed extract was purchased from herbal and medicinal plant store, Benha governorate, Egypt. The drugs were dissolved in normal saline.

\section{Experimental design}

The rats were randomly divided into four groups, 8 rats each:

- group 1: negative control group (normal saline was given daily for 30 days by gastric gavage);

- group 2: the animals were given GSE $(100 \mathrm{mg} /$ $/ \mathrm{kg} / \mathrm{d}$ ) dissolved in normal saline for 30 days by gastric gavage [32]. An amount of $100 \mathrm{mg}$ was dissolved in $10 \mathrm{~mL}$ normal saline and shook to obtain a solution concentration of $10 \mathrm{mg} / \mathrm{mL}$;

- group 3: the animals were given $\mathrm{Cd}(5 \mathrm{mg} / \mathrm{kg} / \mathrm{d})$ dissolved in normal saline for 30 days by gastric gavage [14]. An amount of $5 \mathrm{mg}$ was dissolved in $10 \mathrm{~mL}$ normal saline and shook to obtain a solution concentration of $0.5 \mathrm{mg} / \mathrm{mL}$;

- group 4: the animals were given $\mathrm{Cd}(5 \mathrm{mg} / \mathrm{kg} / \mathrm{d})$ + GSE $(100 \mathrm{mg} / \mathrm{kg} / \mathrm{d})$ for 30 days by gastric gavage. In order to avoid possible drug interactions and drug absorption, the drugs were given in the same time of the day, in two different time intervals. Cadmium was given in the morning (10 AM) while, grape seed extract was given in the afternoon (4 PM). 
Regarding cadmium, the determination of dose and route of administration depended on previous experiments [14], in order to produce target organ toxicity via frequently repeated exposure without induction of animal mortality. The $\mathrm{LD}_{50}$ of $\mathrm{CdCl}_{2}$, anhydrous, when given orally to rats, was reported to be about $88 \mathrm{mg} / \mathrm{kg}$ body weight according to previous work [25], so the dose of cadmium utilised in this study is approximately $1 / 18 \mathrm{LD}_{50}$.

The dose of grape seed extract in experimental animals was $100-400 \mathrm{mg} / \mathrm{kg} / \mathrm{day}$ according to the previously published studies $[2,14,32]$. The selected dose, in the current study was $100 \mathrm{mg} / \mathrm{kg} / \mathrm{d}$ according to the regimen of Sönmez and Tascioglu [32], which was the least effective dose.

At the end of the experiment, animals were euthanized by decapitation under anaesthesia (ketamine + xylazine 75, $10 \mathrm{mg} / \mathrm{kg}$, respectively, IP) and the testes were immediately removed and weighed in each group.

\section{Histological procedures}

Testes were removed from the scrotum, dissected from adherent tissues and weighed. They were fixed in Bouin's solution and processed for paraffin sections. Five-micrometre sections were cut and stained with haematoxylin and eosin (H\&E), based on previously stated protocols [5]. Immunohistochemical staining was done for the detection of expression of proliferating cell nuclear antigen (PCNA) Ab-1, mouse monoclonal antibody (Labvision, Thermo Scientific, USA). The sections were incubated with the primary antibody diluted to a concentration of 1:200 in phosphate buffered saline (PBS) for 1 hour, followed by a reaction with biotinylated secondary antibody. After conjugation with streptavidin-biotin-peroxidase complex, 3,3-diaminobenzidine (DAB) was used as a chromogen, and haematoxylin solution was used as a counterstain. The reaction was nuclear and the positive control was small intestine as shown by Labvision corporation data sheet of PCNA Ab-1. The PCNA immunohistochemistry procedures were done according to the previous protocols [22].

\section{Morphometric study}

The data were obtained using the image analyser computer system (Leica Qwin 500, Leica, England). The image analyser was first calibrated automatically to convert the measurement units (pixels) produced by the image analyser programme into actual mi- crometre units. It was used to measure the area per cent (\%) of PCNA immunoreactions, the diameter of the seminiferous tubules (STs), and the number of normal and abnormal STs. The normal and abnormal STs were evaluated according to their appearance in $\mathrm{H} \& \mathrm{E}-$ stained sections. The normal ones were rounded in transverse section, $150-250 \mu \mathrm{m}$ in diameter, lined by apparently normal stratified spermatogenic epithelium lying on a clear basement membrane and surrounding a narrow lumen. Those that did not show the previously mentioned features were abnormal. For each group, ten measuring fields in each specimen were randomly selected, using low power magnification during the measurement of the diameter of STs, number of the normal STs, and the high power for the area \% of PCNA immunoreaction. Also, the testicular weight of the paired testes of the experimental animals was measured and analysed.

\section{Statistical analysis}

The values obtained were expressed as mean \pm standard deviation for each group. Statistical comparison among different groups was evaluated using one-way analysis of variance (ANOVA) and post hoc LSD test. Calculations were done with SPSS software for windows, version 20. Statistical significance was defined as $p$ value less than 0.05 .

\section{RESULTS}

\section{Mortality and clinical observations}

The dose of cadmium, administered in the current study, caused no mortality among the animals of the different studied groups. No behavioural changes were noticed. Also, no remarkable signs of major system affection were apparent, such as diarrhoea, salivation, oliguria, etc.

\section{Histomorphological results H\&E findings}

Both negative control and GSE-only groups showed a normal testicular architecture with crowded, closely packed STs. Each seminiferous tubule showed apparently normal cellular associations, in the form of stratified arrangement of spermatogenic cells in different stage of developments (spermatogonia, primary and secondary spermatocytes, and spermatids). The spermatogenic cells were seen lying upon a basement membrane occupying most of the tubular thickness. Active releasing spermatozoa were seen within the tubular lumen. Leydig cells and blood cap- 


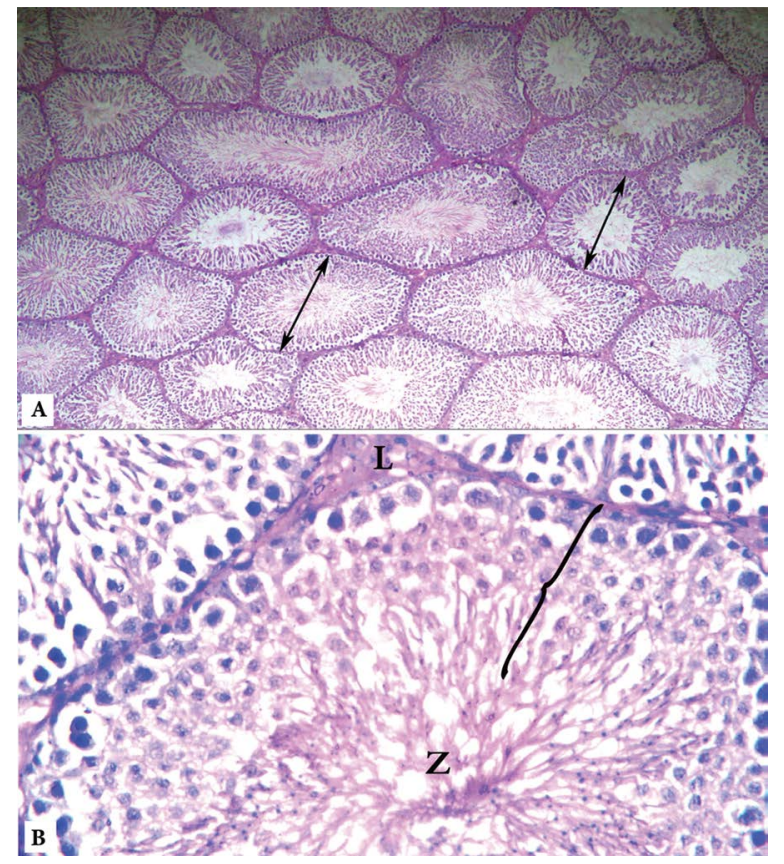

Figure 1. A, B. Haematoxylin and eosin-stained testicular section of a control rat showing crowded, closely packed seminiferous tubules (STs) (double-headed arrows). STs are lined by stratified spermatogenic epithelium (bracket) with releasing spermatozoa (Z) seen within the lumen. The interstitial spaces show Leydig cells $(L)$ (A: $\times 40 ; B: \times 400)$.

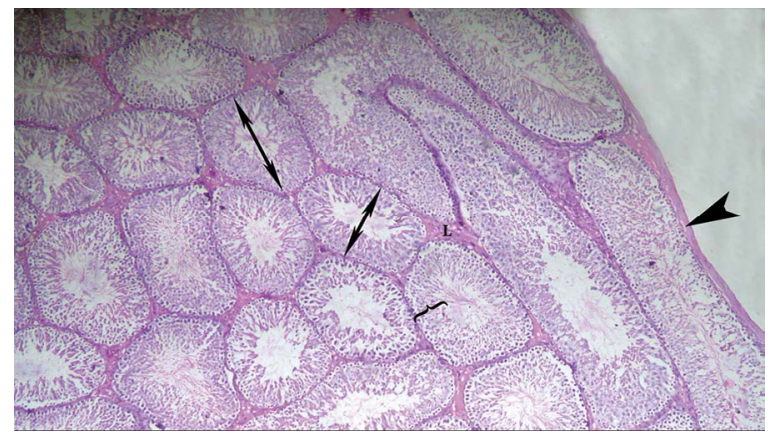

Figure 2. Haematoxylin and eosin-stained testicular section of a rat in grape seed group (group 2) showing numerous packed seminiferous tubules (double-headed arrows) lined by stratified spermatogenic epithelium (bracket) resting on an underlying basement membrane. Interstitial cells of Leydig are seen (L). Tunica albuginea (arrowhead) is seen $(\times 40)$.

illaries were seen in the interstitial spaces, in-between the STs (Figs. 1A, B; 2).

Regarding the administration of $\mathrm{Cd}$ in Cd-onlygroup, all specimens obtained from all animals of the same group showed disorganisation and distortion of the STs. Numerous STs were sloughed and atrophic.
The tunica albuginea was thickened and showed subtunical congestion and oedema (Fig. 3A, B). Degenerated spermatogenic epithelium was seen lining the sloughed STs with thickening of the underlying basement membrane. Only few spermatogonia with many intercellular vacuolations were seen lining the atrophic STs (Fig. 4A-C). Other tubules were severely affected with near-total depletion of their germ cells (Fig. 3C). Pyknotic nuclei of the spermatogenic series were observed (Fig. 3B, C). The interstitial spaces were widened and showed congested blood vessels, accumulation of eosinophilic vacuolated fluid (oedema) and mononuclear cellular infiltrations. The Leydig cells were atrophic, vacuolated or even depleted (Fig. 4A-C).

In Cd + GSE group, the testicular sections obtained from all animals showed relatively normal histoarchitecture, where STs showed more or less normal cellular associations and maintained spermatogenesis. The interstitial cells of Leydig were seen seemingly normal (Fig. 5A, B).

\section{Immunohistochemical observations}

In both negative control and GSE-only groups, positive nuclear PCNA immunoreactivity was observed in the vast majority of the spermatogenic cells of almost all seminiferous tubules (Fig. 6A, B). In Cd group, widely distributed negative PCNA immunoreactivity was noticed in all specimens obtained from all animals of this group (Fig. 6C). Similarly, in $\mathrm{Cd}+$ GSE group, all the obtained specimens showed an amelioration of the positive PCNA immunoreactivity which was limited to the basally located spermatogonia in the vast majority of the STs. However, few STs showed diffuse positive PCNA immunoreaction (Fig. 6D).

\section{Histomorphometric evaluation of the area per cent of PCNA immunoreactivity}

The statistical data, summarised in Table 1, revealed that the mean area \% of PCNA immunostaining in $\mathrm{Cd}$ group was significantly lower $(p<0.05)$ when compared to control group and GSE-only group. However, no significant difference was present in GSE-only group compared to control group. In Cd + GSE group, the mean area \% of PCNA immunostaining was significantly higher $(p<0.05)$ compared to $\mathrm{Cd}$ group. Comparing to either the control or GSE-only groups, the mean area \% of PCNA immunoexpression was significantly lower $(p<0.05)$. 


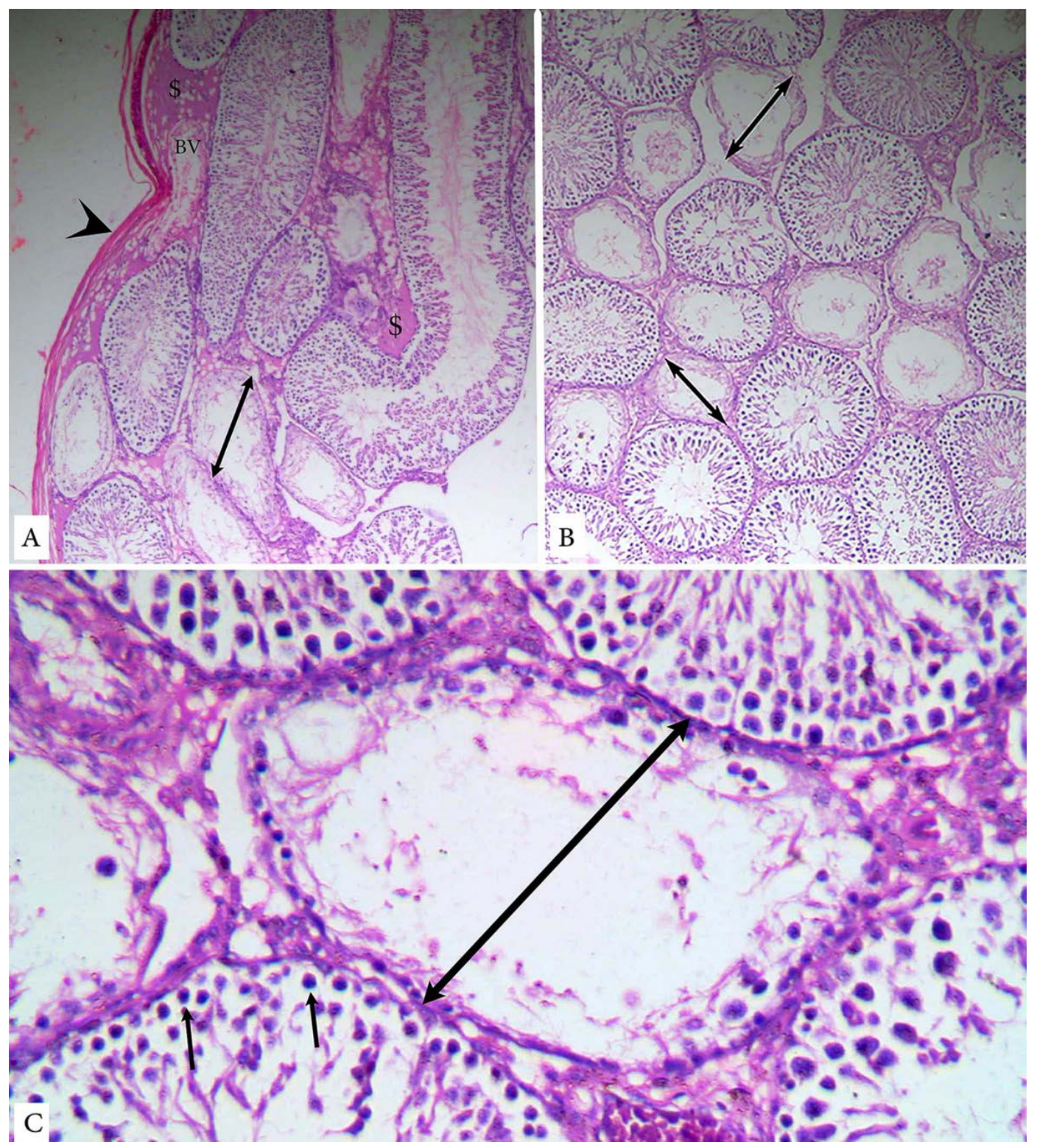

Figure 3. A, B, C. Haematoxylin and eosin-stained testicular section of a rat in the cadmium group (group 3) showing disorganised widely spaced seminiferous tubules (STs). Numerous STs (double-headed arrows) are atrophic and sloughed with nearly totally depleted germ cells. The spermatogenic cells show pyknotic nuclei (short arrows). The interstitial spaces show eosinophilic vacuolated fluid (oedema) (dollar sign) and congestion (BV). Tunica albuginea (arrowhead) is thickened and show subtunical congestion (BV) and oedema (dollar sign) $(\mathrm{A}, \mathrm{B}: \times 40 ; \mathrm{C}: \times 200)$.

\section{Histomorphometric evaluation of the diameter of the STS}

As shown in Table 1, the mean diameter of the STs in Cd group was significantly lower when compared to control rats. No significant difference was observed in GSE-only group when compared to group 1. In $\mathrm{Cd}+$ GSE group, the mean diameter of STs was significantly higher when compared to Cd group, but no difference of statistical significance was present when compared to control and GSE groups.

\section{Histomorphometric evaluation of the per cent of the normal STs}

Similarly, the per cent of the normal STs was significantly lower in Cd group when compared to control rats. No significant difference was observed in GSE-only group when compared to the control. In $\mathrm{Cd}+\mathrm{GSE}$ group, the per cent of the normal STs was significantly higher when compared to Cd group, but still significantly lower when compared to control group (Table 1). 

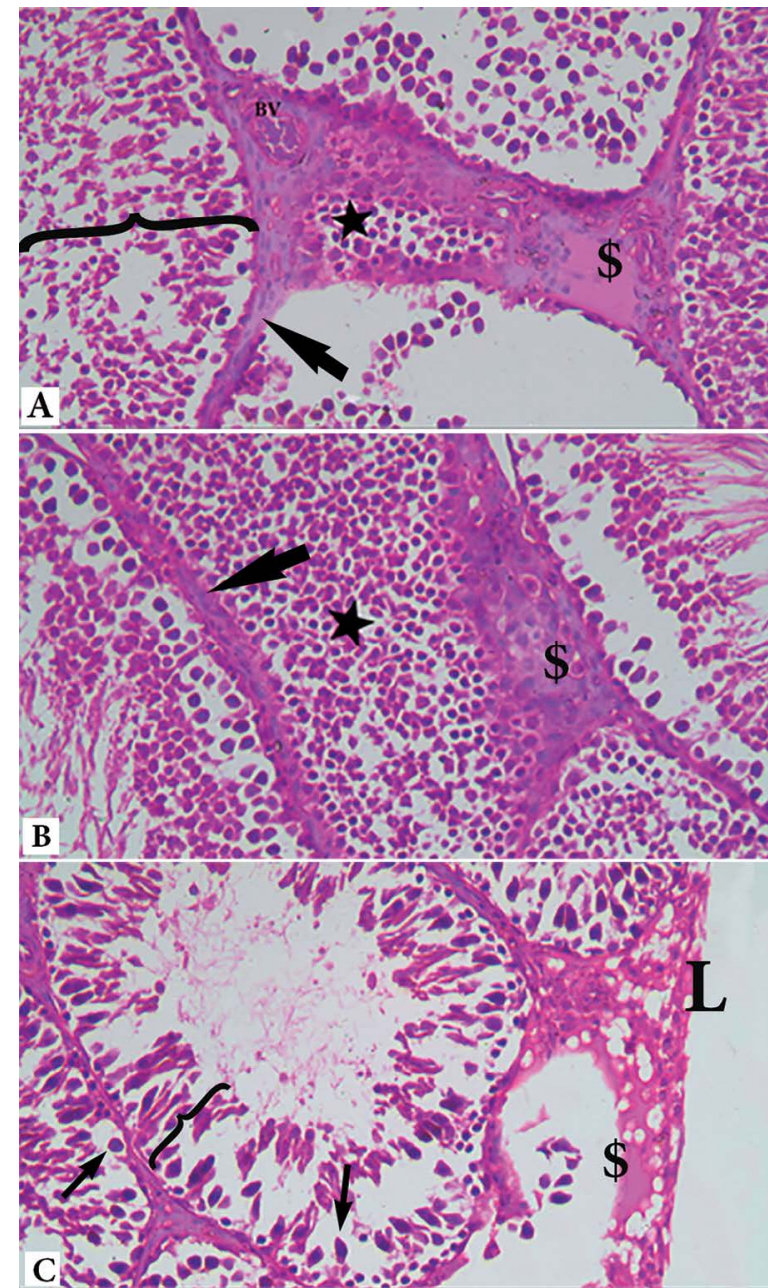

Figure 4. A, B, C. Haematoxylin and eosin-stained testicular section of a rat in the cadmium group (group 3 ) showing degenerated spermatogenic epithelium (brackets) and loss of the releasing spermatozoa. The majority of seminiferous tubules show sloughing and numerous intraepithelial empty spaces (vacuolations) and cellular detachment. Pyknotic nuclei of the spermatogenic cell are seen (short arrows). The peritubular basement membrane (thick arrows) is thickened with numerous flat myoid cells. The interstitial space is widened and shows accumulation of eosinophilic vacuolated fluid accumulation (oedema) (dollar signs) and infiltration of mononuclear cells (stars). Leydig cells (L) are more or less small sized and vacuolated $(A, B, C: \times 200)$.

\section{Testicular weight}

Table 1 showed that the mean weight of the paired testes in $\mathrm{Cd}$ group was significantly lower when compared to control rats and GSE-only group. No significant difference was present in GSE-only group compared to control group. In Cd + GSE group, the mean testicular weight was significantly higher when compared to $\mathrm{Cd}$ group but still significantly lower than that of control and GSE groups.
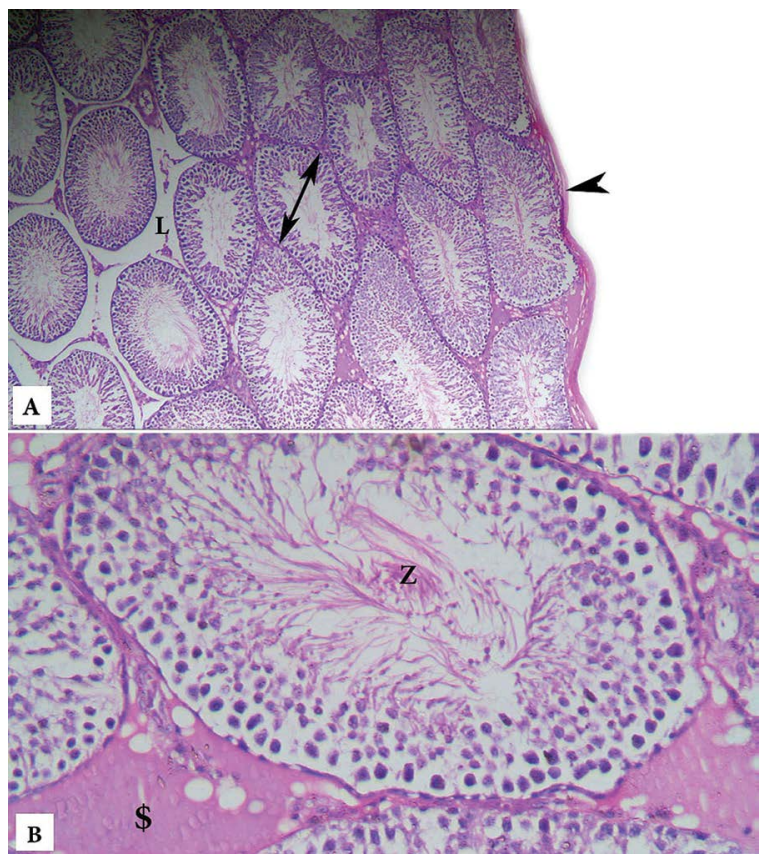

Figure 5. A, B. Haematoxylin and eosin-stained testicular section of a rat in cadmium + grape seed group (group 4) showing normal shaped seminiferous tubules (double-headed arrow) with more or less normal cellularity. The releasing spermatozoa (Z) are seen within the lumen. The interstitial space is wide and shows accumulation of eosinophilic vacuolated fluid (dollar sign). Leydig cells (L) are seen. The tunica albuginea (arrowhead) appears more or less normal $(A: \times 40 ; B: \times 200)$.

\section{DISCUSSION}

Cadmium is a highly toxic heavy metal and has spread widely in the environment in recent decades due to its varied industrial applications [17]. The current study aimed at studying the $\mathrm{Cd}$-induced testicular histopathological alterations and its counteracting and amelioration by GSE.

The present work showed that oral cadmium $\left(\mathrm{CdCl}_{2}\right)$ administration in rats (group 3 ) induced major structural alterations in the testis, which were ameliorated by the administration of GSE in group 4 . $\mathrm{CdCl}_{2}$ toxicity produced significant structural changes in the testis in the form of distortion of the STs, with cellular disorganisation, oedema, and sloughing of the seminiferous epithelium which was accumulated in the centre of the STs in the form of degenerated tissue. Many STs showed widely spaced spermatogenic cells and decreased sperm count. These results were consistent with that of other investigators [26] who reported a similar finding. Active spermatogenesis was affected which was denoted by the absence or 

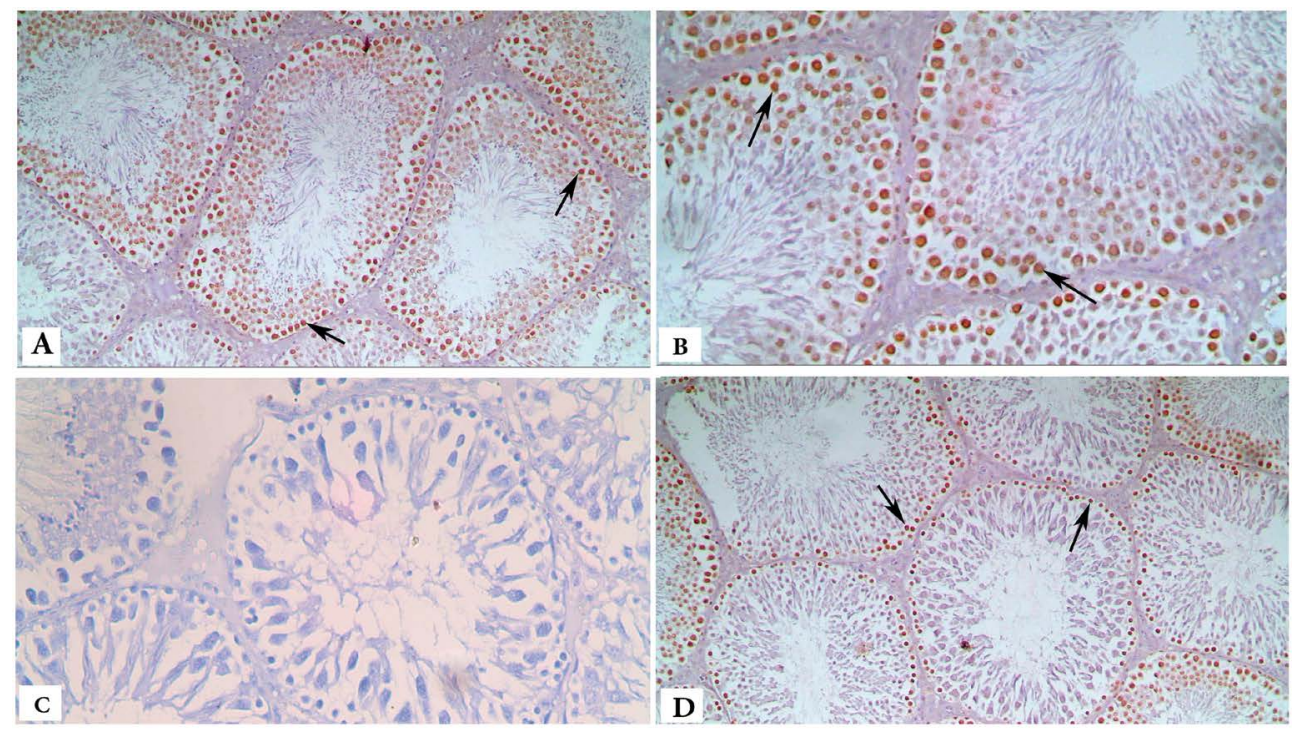

Figure 6. Proliferating nuclear cell antigen (PCNA) immunostained photomicrographs of testicular sections taken from control rats (A), group 2 (B), group 3 (C) and group 4 (D). Marked positive PCNA immunostaining of nuclei (arrows) of all the spermatogenic series is evident in groups 1 and 2 while, limited to the basally located spermatogonia (arrows) in group 4. Negative nuclear PCNA immunoreactivity is observed in group 3 (PCNA immunostaining, $A, D: \times 100 ; B, C: \times 200$ ).

Table 1. Different histomorphometric parameters and testicular weight [g] of control, grape seed extract (GSE), cadmium (Cd), Cd + GSE groups

\begin{tabular}{lcccc}
\hline & Control group (G1) & GSE group (G2) & Cd group (G3) & Cd +GSE group (G4) \\
\hline Area per cent of PCNA & $21.21 \pm 1.81$ & $21.08 \pm 1.79$ & $0.69 \pm 1.26^{\mathrm{a}}$ & $17.09 \pm 1.26^{\mathrm{b}}$ \\
Diameter of STs $[\mu \mathrm{m}]$ & $243.70 \pm 9.18$ & $243 \pm 8.81$ & $178.5 \pm 12.02^{\mathrm{a}}$ & $235.61 \pm 9.30^{\mathrm{c}}$ \\
Per cent of the normal STs & $88 \pm 5.75$ & $87.7 \pm 5.31$ & $19.4 \pm 2.88^{\mathrm{a}}$ & $76.9 \pm 4.12^{\mathrm{b}}$ \\
Testicular weight $[\mathrm{g}]$ & $3.07 \pm 0.18$ & $3.05 \pm 0.17$ & $1.5 \pm 0.12^{\mathrm{a}}$ & $2.43 \pm 0.15^{\mathrm{b}}$ \\
\hline
\end{tabular}

The data were expressed as mean \pm standard deviation $(n=8)$. a Significantly different when compared to $\mathrm{G} 1$ and $\mathrm{G} 2$; ' Significantly different when compared to $\mathrm{G} 3$ and $\mathrm{G} 1$ and $\mathrm{G} 2$; 'Significantly different when compared to G3, using post hoc ANOVA (LSD), p value $<0.05$; PCNA — proliferating nuclear cell antigen; STs - seminiferous tubules

decreasing of the releasing spermatozoa within the STs lumen.

In agreement with the results of another study [13], the present work showed widening of interstitial spaces due to vascular congestion, diffuse infiltrations of mononuclear cells, and accumulation of eosinophilic vacuolated fluid (oedema) in-between the STs. Moreover, the results of the current study showed pyknosis of the nuclei of the spermatogenic series in $\mathrm{Cd}$ group (group 3 ) which was similar to the findings of other authors [12] who studied the toxic effects of $\mathrm{Cd}$ on different organ systems of rodents and revealed pyknotic nuclear changes, particularly in the testis.

Furthermore, many STs showed marked atrophy with degeneration and loss of the seminiferous epithelium, which was confirmed by the mean diameter of STs and the per cent of the normal STs. The mean tubular diameter and the per cent of the normal STs in $\mathrm{Cd}$ group were significantly lower when compared to that of the control rats. These results were in accordance with other authors [1] who reported atrophy of the seminiferous epithelium and decrease in the tubular lumen $36 \mathrm{~h}$ after intraperitoneal $\mathrm{Cd}$ injection in rats. In Cd + GSE group, both the mean tubular diameter and the per cent of the normal STs were markedly high, which was significantly different when compared to Cd group. No statistical significance was present comparing to control or GSE-only rats, which means complete maintaining of the tubular diameter but still the number of the normal STs.

The atrophy and degeneration of the STs may be the cause of weight loss of the testes of $\mathrm{Cd}$ intoxicated rats 
(group 3). The mean testicular weight of the animals of group 3 was significantly lower when compared with other groups. This finding was parallel to that of another study [25] which revealed a marked decrease in the testicular weight 28 days after oral $\mathrm{Cd}$ administration in rats. In Cd + GSE group, the mean testicular weight was significantly higher when compared to $\mathrm{Cd}$ group, but still significantly lower than those in control and GSE groups. This means that the co-administration of GSE with Cd, in group 4 attenuated the Cd-induced histopathological changes in the testes and hence had fewer effects on the testicular weight.

Cadmium toxicity has been encountered in several organs such as kidney, brain, pancreas, blood, and immune system [6]. Cd-induced testicular damage was dose-dependent. Low dose $\mathrm{Cd}$ affects no organs other than the testis [10]. The underlying mechanism of testicular toxicity remains obscure. One explanation supposed that $\mathrm{Cd}$ toxicity induced vascular damage, mainly in the testis, which may result in the inability to metabolise zinc, with its subsequent replacement by $\mathrm{Cd}$ in the testicular blood circulation $[3,27]$. The dose utilised in this study was optimal to induce testicular toxicity without lethal effects.

The previously mentioned structural alterations in the testis could be explained by the accumulation of cadmium in different organs, and hence the testis [16] where it caused testicular damage. The accumulated $\mathrm{Cd}$ stimulated the overproduction of reactive oxygen species through depletion of the reduced glutathione and reacting with the sulfhydryl groups of proteins. Furthermore, Cd inhibited the activity of antioxidant enzymes such as catalase and superoxide dismutase [28]. Subsequently, the resulting oxidative stress induced DNA fragmentation and apoptotic changes as reported by other authors [34].

In the Cd + GSE treated rats, marked improvement was noticed in the testes, in the form of good organisation of the STs and more or less normal cellular associations of the seminiferous epithelium. This protective effect of GSE might be attributed to not only its potential effects against oxidative stress but also the inhibition of the free radical production with subsequent maintaining of the affected antioxidant protection system. This explanation was in agreement with that of other authors [11].

Regarding the immunohistochemical results, PCNA is a nuclear protein, used as a marker for cell proliferation [19]. So, the detection of any PCNA immunostained spermatogenic cell refers to the activity and the efficiency of spermatogenesis. Administration of $\mathrm{Cd}$ to the animals of the current study (in group 3) revealed very few positive immunostained cells for PCNA or even negative in most of the testicular sections, which was significant when compared to the control group. These findings could refer to that the oxidative damage induced in the testis by $\mathrm{Cd}$ is associated with depletion of the active DNA contents in the dividing spermatogenic cells. No significant changes were detected in the rat group that received grape seed extract alone. However, the co-administration of grape seed extract (in group 4) showed a significantly higher number of PCNA immunoreactive spermatogenic cells, but still significantly lower than those in control and GSE groups. These results were consistent with the finding of the other authors [2] who reported similar results when they used Ki 67 as a marker of proliferation.

The results of the current study were parallel to that of Sönmez and Tascioglu [32] who reported an increase in the number of immunostained apoptotic cells and hence, decrease in the number of the proliferating cells in $\mathrm{Cd}$ intoxicated rats which were protected against by GSE.

When correlating the results of the PCNA immunoreactivity and the mean testicular weight in $\mathrm{Cd}+$ GSE group (group 4), both parameters were significantly higher compared to Cd group, but still significantly lower than those of control and GSE groups. This means that the GSE could protect against Cd-induced testicular alterations which had fewer effects on testicular weight and on the proliferative activity of spermatogenic cells, as confirmed by PCNA immunohistochemistry.

In human studies, there was a wide safety margin of the grape seed dosage. The adjustment of the dose was effect-dependent. The dose was variable according to the indicated usage i.e. antioxidant, anti-inflammatory, blood capillary improving effect, etc. [20]. In a safety assessment study [29] of 4-week oral intake of GSE in healthy individuals, a dose up to $2500 \mathrm{mg}$ was reported to be safe.

\section{Limitations and areas for future research}

There are two major limitations in the current study that could be addressed in future research. First, the study focused mainly on the microscopic structure of the testes of the experimental animals. If more resources had been available; we would have studied the correlation of the testicular struc- 
ture with other parameters like oxidative status and the endocrine and exocrine functions of the testis. However, this study was self-funded without any external support.

Second, given the cross-sectional design that we have used, the current study was not conclusive regarding the therapeutic effects of grape seed extract on the testicular histopathological alterations induced by $\mathrm{Cd}$ toxicity. Future research should aim at a more comprehensive assessment of $\mathrm{Cd}$ toxicity on the hormonal, reproductive, and oxidative status of the testes. This assessment could be based on the measurement of the hormonal profile e.g. serum testosterone, follicular stimulating hormone, and luteinizing hormone, evaluation of semen parameters via epididymal semen analysis, and measurement of the testicular level of oxidative enzymes like superoxide dismutase. Needless to say that a more comprehensive study design is needed to assess the therapeutic effects of grape seed extract.

\section{CONCLUSIONS}

Finally, administration of grape seed extract reduced the histopathological testicular changes induced by cadmium chloride. This effect may be related to the antioxidant property of grape seed extracts as confirmed by maintaining the histological architecture of the testes and retaining of the proliferative activity of the spermatogenic cells. The potential antioxidant property of GSE recommends its use in those men with known high risk of cadmium exposure, such as workers in battery factories, to protect against the oxidative stress elicited in the testes due to chronic asymptomatic occupational exposure.

\section{Acknowledgements}

Great consideration and deep gratitude were expressed to all our colleagues in the Histology Departments, Faculties of Medicine in Benha and Fayoum Universities for their support. Special thanks to Dr. Ghaiath Hussien who has read the article and revised it for further editing.

\section{REFERENCES}

1. Adamkovičová M, Toman R, Cabaj M. Diazinon and cadmium acute testicular toxicity in rats examined by histological and morphometrical methods. 2010; 2010(3): 134-140.

2. Alkhedaide A, Alshehri ZS, Sabry A, et al. Protective effect of grape seed extract against cadmium-induced testicular dysfunction. Mol Med Rep. 2016; 13(4): 3101-3109, doi: 10.3892/mmr.2016.4928, indexed in Pubmed: 26935153.
3. Amara S, Abdelmelek H, Garrel C, et al. Preventive effect of zinc against cadmium-induced oxidative stress in the rat testis. J Reprod Dev. 2008; 54(2): 129-134, doi: 10.1262/ jrd.18110, indexed in Pubmed: 17420618.

4. Faroon O, Ashizawa A, Wrigh S, et al. Toxicological Profile for Cadmium. U.S. Department of Health and Human Services, Public Health Service, Atlanta, Ga, USA. 2012.

5. Bancroft JD, Lyton C. The Hematoxylins and Eosin. In: Suvarna SK, eds. Theory and Practice of Histological Techniques. 8th ed. 2018: 126-138.

6. Bernhoft RA. Cadmium toxicity and treatment. Sci World J. 2013; 2013: 394652, doi: 10.1155/2013/394652, indexed in Pubmed: 23844395.

7. Blanco A, Moyano R, Vivo J, et al. Quantitative changes in the testicular structure in mice exposed to low doses of cadmium. Environ Toxicol Pharmacol. 2007; 23(1): 96-101, doi: 10.1016/j.etap.2006.07.008, indexed in Pubmed: 21783742.

8. Burukoğlu D, Bayçu C. Protective effects of zinc on testes of cadmium-treated rats. Bull Environ Contam Toxicol. 2008; 81(6): 521-524, doi: 10.1007/s00128-007-9211-x, indexed in Pubmed: 18925378.

9. Committee NRC. Guide for the Care and Use of Laboratory Animals: NIH Publications No. 8023. 8th ed. National Academies Press; 2010; 1-220.

10. de Souza Predes F, Diamante MA, Dolder H. Testis response to low doses of cadmium in Wistar rats. Int J Exp Pathol. 2010; 91(2): 125-131, doi: 10.1111/j.13652613.2009.00692.x, indexed in Pubmed: 20015210.

11. El-Beshbishy $H$, Mohamadin A, Abdel-Naim A. In Vitro Evaluation of the Antioxidant Activities of Grape Seed (Vitis vinifera) Extract, Blackseed (Nigella sativa) Extract and Curcumin. J Taibah Univ Med Sci. 2009; 4(1): 23-35, doi: 10.1016/s1658-3612(09)70078-2.

12. El-Refaiy Al, Eissa FI. Histopathology and cytotoxicity as biomarkers in treated rats with cadmium and some therapeutic agents. Saudi J Biol Sci. 2013; 20(3): 265-280, doi: 10.1016/j.sjbs.2013.02.004, indexed in Pubmed: 23961244.

13. El-Shahat AR, Gabr A, Meki AR, et al. Altered Testicular Morphology and Oxidative Stress Induced by Cadmium in Experimental Rats and Protective Effect of Simultaneous Green Tea Extract. Int J Morphol. 2009; 27(3), doi: 10.4067/s0717-95022009000300020.

14. Evcimen $M$, Demirel $H H$, Aslan $R$, et al. For rats which are implemented cadmium chloride exposure, polydatin and grape seed extracts protective effcts on testis and brain tissues. Glob J Res Anal. 2015; 4(10): 307-310, doi: 10.15373/22778160.

15. Filippini T, Cilloni S, Malavolti M, et al. Dietary intake of cadmium, chromium, copper, manganese, selenium and zinc in a Northern Italy community. J Trace Elem Med Biol. 2018; 50: 508-517, doi: 10.1016/j.jtemb.2018.03.001, indexed in Pubmed: 29548610.

16. Godt J, Scheidig F, Grosse-Siestrup C, et al. The toxicity of cadmium and resulting hazards for human health. J Occup Med Toxicol. 2006; 1: 22, doi: 10.1186/1745-6673-1-22, indexed in Pubmed: 16961932.

17. Huang $Y$, He C, Shen $C$, et al. Toxicity of cadmium and its health risks from leafy vegetable consumption. Food Funct. 2017; 8(4): 1373-1401, doi: 10.1039/c6fo01580h, indexed in Pubmed: 28232985. 
18. Jia Z, Song Z, Zhao Y, et al. Grape seed proanthocyanidin extract protects human lens epithelial cells from oxidative stress via reducing NF-small ka, CyrillicB and MAPK protein expression. Molecuar Vis. 2011; 17: 210-217, indexed in Pubmed: 21264233.

19. Juríková M, Danihel L', Polák Š, et al. Ki67, PCNA, and MCM proteins: Markers of proliferation in the diagnosis of breast cancer. Acta Histochem. 2016; 118(5): 544-552, doi: 10.1016/j.acthis.2016.05.002, indexed in Pubmed: 27246286.

20. Kappagoda CT, Burton-Freeman B, Edirisinghe I. Modulation of oxidative stress, inflammation, and impaired insulin sensitivity with grape seed extract. June 2017.

21. Kaur M, Agarwal C, Agarwal R. Anticancer and cancer chemopreventive potential of grape seed extract and other grape-based products. J Nutr. 2009; 139(9): 1806S-1812S, doi: 10.3945/jn.109.106864, indexed in Pubmed: 19640973.

22. Kiernan JA. Immunohistochemistry. In: Kiernan J, ed. Histological and Histochemical Methods. Theory and Practice. 4th ed. Scion Publishing Ltd 2015: 454-490.

23. Mandic A, Đilas S, Ćetković G, et al. Polyphenolic Composition and Antioxidant Activities of Grape Seed Extract. Int J Food Prop. 2008; 11(4): 713-726, doi: 10.1080/10942910701584260.

24. Narges K, Tavakkoli L. Environmental and occupational exposure to cadmium in Iran: a systematic review. Rev Environ Health. 2016; 31(4): 457-463, doi: 10.1515/ reveh-2016-0042, indexed in Pubmed: 27902453.

25. Onwuka FC, Erhabor O, Eteng MU, et al. Ameliorative effect of cabbage extract on cadmium- induced changes on hematology and biochemical parameters of albino rats. J Toxicol Environ Heal Sci. 2010; 2(2): 11-16.

26. Pires VC, Gollücke AP, Ribeiro DA, et al. Grape juice concentrate protects reproductive parameters of male rats against cadmium-induced damage: a chronic assay. Br J Nutr. 2013; 110(11): 2020-2029, doi: 10.1017/ S0007114513001360, indexed in Pubmed: 23656754.
27. Prozialeck WC, Edwards JR, Nebert DW, et al. The vascular system as a target of metal toxicity. Toxicol Sci. 2008; 102(2): 207-218, doi: 10.1093/toxsci/kfm263, indexed in Pubmed: 17947343.

28. Rahimzadeh MR, Rahimzadeh MR, Kazemi S, et al. Cadmium toxicity and treatment: An update. Casp J Intern Med. 2017; 8(3): 135.

29. Sano A. Safety assessment of 4-week oral intake of proanthocyanidin-rich grape seed extract in healthy subjects. Food Chem Toxicol. 2017; 108(Pt B): 519-523, doi: 10.1016/j.fct.2016.11.021, indexed in Pubmed: 27889390.

30. Sehirli O, Ozel Y, Dulundu E, et al. Grape seed extract treatment reduces hepatic ischemia-reperfusion injury in rats. Phytother Res. 2008; 22(1): 43-48, doi: 10.1002/ ptr.2256, indexed in Pubmed: 18165941.

31. Siu ER, Mruk DD, Porto CS, et al. Cadmium-induced testicular injury. Toxicol Appl Pharmacol. 2009; 238(3): 240-249, doi: 10.1016/j.taap.2009.01.028, indexed in Pubmed: 19236889.

32. Sönmez MF, Tascioglu S. Protective effects of grape seed extract on cadmium-induced testicular damage, apoptosis, and endothelial nitric oxide synthases expression in rats. Toxicol Ind Health. 2016; 32(8): 1486-1494, doi: 10.1177/0748233714566874, indexed in Pubmed: 25614582.

33. Terra $X$, Pallarés $V$, Ardèvol $A$, et al. Modulatory effect of grape-seed procyanidins on local and systemic inflammation in diet-induced obesity rats. J Nutr Biochem. 2011; 22(4): 380-387, doi: 10.1016/j.jnutbio.2010.03.006, indexed in Pubmed: 20655715.

34. Wang L, Xu T, Lei W, et al. Cadmium-induced oxidative stress and apoptotic changes in the testis of freshwater crab, Sinopotamon henanense. PLoS One. 2011; 6(11).

35. WHO. Exposure to Cadmium: A Major Public Health Concern; 2010.

36. $\mathrm{Xu} X, \mathrm{Nie} S$, Ding $\mathrm{H}$, et al. Environmental pollution and kidney diseases. Nat Rev Nephrol. 2018; 14(5): 313-324, doi: 10.1038/nrneph.2018.11, indexed in Pubmed: 29479079. 